\title{
Editoriale
}

\section{Nefropatia da mezzodi contrasto: il parere del Nefrologo}

\author{
A. Lacquaniti e M. Buemi
}

Cattedra di Nefrologia, Università di Messina, Messina

CONTRAST-INDUCED NEPHROPATHY: NEPHROLOGIST'S OPINION

AвStRact. Contrast-induced nephropathy (CIN) has become the third leading cause of hospital-acquired acute kidney injury (AKI), due to an increasing number of patients receiving intravascular injection of iodinated contrast media (ICM) every year worldwide.

The risk for patients with predisposing factors, such as advanced age, pre-existing renal disease, congestive heart failure, anterior myocardial infarction, cardiogenic shock, diabetes mellitus and type and volume of contrast, is considerably increased and ranges between approximately $12 \%$ and $50 \%$.

Among the contemporary definitions, CIN is characterized by an absolute $(\geq 0.5 \mathrm{mg} / \mathrm{dL})$ and relative increase $(\geq 25 \%)$ in serum creatinine (sCreat) at 48-72 hours after exposure to a contrast agent compared to baseline sCreat values, when alternative explanations for renal impairment have been excluded.

In patients with acute changes in their glomerular filtration rate (GFR), sCreat is a relatively inaccurate marker, and its alterations are not particularly sensitive or specific for small changes in the GFR. In fact, $25-50 \%$ of the increase in creatinine, which is highly predictive for the development of CIN, most often occurs only after 24 hours after administration of ICM.

In the last decade, it has been conducted studies not only for the early detection of biomarkers, but also to assess several therapeutic strategies to prevent CIN.

A widely accepted prophylaxis for CIN after CM application is based on an intravenous volume load with an isotonic saline infusion $(0.9 \%)$.

Hydration with sodium bicarbonate should provide additional renal protection by alkalinizing renal tubular fluid, with a decreased tubular damage by scavenging oxygen free radicals.

$\mathrm{N}$-acetylcysteine (NAC) has been the most widely studied of all prophylaxis strategies.

Unfortunately, most studies lacked statistical power, used different types of CM, various definitions of CIN and several diverse prophylactic measures. This resulted in the lack of accepted guidelines among radiologists, cardiologists, nephrologists and other involved physicians.

Studies will be designed to clarify the value of these preventive therapies, analysing new indicators of renal function such as NGAL or Cistatin C.

KEY WoRDs. CIN, AKI, Iodinated contrast media, GFR

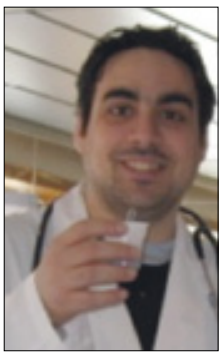

Antonio Lacquaniti
Negli ultimi anni la medicina ha subito una rivoluzione grazie all'introduzione di nuove procedure di diagnostica per immagini e al perfezionamento di quelle già esistenti. Queste hanno apportato informazioni non comparabili al tradizionale esame semeiotico, permettendo così, caso per caso, la formulazione di un più preciso ed accurato indirizzo diagnostico, terapeutico e prognostico. Tuttavia, di pari passo con lo sviluppo incoraggiante di queste procedure, si è reso necessario affrontare il pro- blema inerente gli effetti avversi delle stesse, correlati, nella maggior parte dei casi, alla somministrazione di sostanze chimiche utilizzate per enfatizzare la visione della patologia. Ovviamente non tutte le sostanze impiegate nella diagnostica per immagini sono da considerare nefrotossiche, ma i possibili danni che possono indurre nel rene devono allertare non solo il clinico, che richiede esami diagnostici, ma anche il radiologo. Da questo punto di vista la piena comunicazione tra queste due figure è un requisito fondamentale al fine di evitare una temibile complicanza renale quale la nefropatia da mezzo di contrasto (CIN). 
La CIN rappresenta oggi la terza causa di insufficienza renale acuta (AKI) in pazienti ospedalizzati (1). Ciò è da ricondurre a un aumentato numero di soggetti che vengono sottoposti annualmente a procedure radiologiche interventistiche richiedenti la somministrazione intravascolare di mezzi di contrasto $(\mathrm{MdC})$ iodati e dall'incremento dei fattori di rischio che caratterizzano questi pazienti.

Siamo infatti di fronte a soggetti di età avanzata, aventi una preesistente nefropatia, affetti da cardiopatia congestiva o con pregresso infarto del miocardio, diabetici e che assumono una serie di farmaci spesso nefrotossici. Logicamente il tipo di MdC utilizzato e la dose somministrata giocano un ruolo chiave nel processo fisiopatologico della CIN.

Esistono diversi tipi di MdC, con le sostanze iodate maggiormente utilizzate, in quanto lo iodio è alla base della radiopacità, ma purtroppo strettamente connesse allo sviluppo di CIN.

Il gadolinio, inizialmente considerato non nefrotossico, è stato utilizzato negli ultimi anni come valido sostituto dei MdC iodati in pazienti a rischio di CIN. Di sicuro un $\mathrm{MdC}$ non innocuo visto che si è registrato un incremento notevole della prevalenza di casi di CIN dopo somministrazione di gadolinio, specie in pazienti con una insufficienza renale avanzata.

Non sono ben definiti i meccanismi di nefrotossicità, ma sicuramente la dose con cui viene somministrato e il grado di nefropatia preesistente sono elementi fondamentali per l'esordio del danno renale acuto. Anche se negli ultimi anni sono stati effettuati numerosi studi al fine di individuare l'esatto meccanismo della CIN, numerosi sono i fattori patogenetici potenziali oggi chiamati in causa, non escludentesi a vicenda ma, anzi, singoli elementi di un puzzle che rendono questa patologia molto complessa.

Questo progresso nello studio della fisiopatologia della CIN non si è però concretizzato in marcatori precoci in grado di identificare una CIN. Oggi la sua diagnosi viene effettuata attraverso un "marcatore" dotato di bassissima sensibilità e specificità quale la creatinina. Tutte le definizioni che appaiono su riviste internazionali nefrologiche e radiologiche si basano sulle sue variazioni registrate nell'arco di 48-72 ore dalla esposizione del contrasto. Un tempo a dir poco infinito, considerando che i cardiologi sono dotati di marcatori in grado di svelare anche una lieve sofferenza cardiaca dopo solo pochi minuti, e non dopo giorni.

È noto come la creatinina non sia in grado di rispecchiare condizioni cliniche caratterizzate da rapide e acute variazioni del filtrato glomerulare renale, e la
CIN ne è un esempio. Non è un caso che oggi le definizioni di questa condizione si basino su un così lungo periodo osservazionale. Inoltre si registra il picco di incidenza di CIN solo dopo 24 ore dalla somministrazione del $\mathrm{MdC}$, e non prima, perché non abbiamo test diagnostici a disposizione per rilevarla.

Marcatori promettenti sono comunque alla porta. Kidney injury molecule-1 (KIM-1) e la Cistatina C si positivizzano dopo $12-24$ ore circa dalla sommnistrazione del $\mathrm{MdC}$ mentre un altro promettente marcatore, Neutrophil-Gelatinase associated lipocalin (NGAL) si positivizza dopo appena 2 ore (2). Non saranno forse la "troponina" del rene, ma ulteriori studi sono necessari al fine di validare un marcatore o un pannello di marcatori per una diagnosi ottimale di CIN.

Altro aspetto dibattuto è la scelta terapeutica preventiva da dover effettuare in pazienti che devono sottoporsi a procedure diagnostiche e/o interventistiche richiedenti l'uso del MdC.

Una terapia accettata è la somministrazione di soluzione fisiologica allo $0.9 \%$.

Una "semplice" idratazione determinerebbe infatti una serie di azioni preventive a livello renale, attraverso una soppressione del sistema renina-angiotensina-aldosterone con una conseguente aumentata escrezione di sodio da parte del tubulo distale del nefrone, una down regulation del feedback tubuloglomerulare, una diluizione del $\mathrm{MdC}$ e una ridotta vasocostrizione a livello della corticale renale (3).

Altra scelta terapeutica si basa sulla somministrazione di bicarbonato di sodio, in grado di alcalinizzare le urine e ridurre il danno mediato dai radicali liberi dell'ossigeno.

Infine, oggetto di molti studi sulle strategie preventive della CIN è stata l'N-acetilcisteina (4).

Diversi sono i meccanismi chiamati in causa, tra cui una vasodilatazione a livello renale con conseguente miglioramento dell'emodinamica locale, una riduzione della disfunzione endoteliale, una prevenzione diretta del danno tissutale di tipo ossidativo mediato dai radicali liberi dell'ossigeno.

$\mathrm{Ma}$ anche in questo ambito, così come abbiamo visto nel contesto della diagnosi della CIN, vi sono alternanze di dubbi e certezze. La maggior parte dei trials mancano di potenza statistica, utilizzano diversi tipi di MdC, e varie definizioni di CIN e troppi schemi terapeutici preventivi vengono utilizzati. Questo è la base, per cui non esistono linee guida universalmente accettate dai nefrologi, radiologi o cardiologi ma come sempre è il medico che caso per caso deve "decidere" davanti al quadro clinico che il paziente presenta. 


\section{Riassunto}

La nefropatia da contrasto (CIN) rappresenta oggi la terza causa di insufficienza renale acuta (AKI) in pazienti ospedalizzati, condizione da ricondurre a un incremento dei pazienti che si sottopongono a procedure radiologiche interventistiche richiedenti la somministrazione intravascolare di mezzi di contrasto iodati (ICM).

Bisogna inoltre considerare un incremento di soggetti con fattori di rischio quali l'età avanzata, una preesistente patologia renale, scompenso cardiaco, infarto del miocardio, diabete mellito.

Si considera CIN la presenza di un incremento assoluto ( $\geq 0.5 \mathrm{mg} / \mathrm{dL}$ ) e relativo ( $\geq 25 \%$ ), rispetto ai valori basali, della creatinina sierica (sCreat) a 48-72 ore dall'esposizione dell'ICM.

È noto però come in pazienti con variazioni acute del filtrato glomerulare (GFR), sCreat è un marker dotato di poca sensibilità e specificità diagnostica. Infatti, il 25-50\% dell'incremento della creatinina, con alto valore predittivo di CIN, si verifica più frequentemente solo 24 ore dopo la somministrazione dell'ICM.

Negli ultimi anni, sono stati condotti studi non solo al fine di identificare nuovi biomarcatori, ma anche per valutare eventuali strategie terapeutiche preventive.

La somministrazione endovenosa di soluzione salina allo $0.9 \%$ è ampiamente accettata come terapia profilattica di CIN. Diversi sono inoltre gli studi condotti che prevedono la somministrazione di bicarbonato di sodio o di $\mathrm{N}$-acetilcisteina (NAC). Purtroppo molti studi mancano di potenza statistica o sono basati su diverse definizioni di CIN. Ciò ha determinato la mancanza di linee guida universali accettate dai radiologi, nefrologi, cardiologi o da altre figure professionali coinvolte.

Sono quindi necessari ulteriori studi al fine di validare i risultati sino ad ora ottenuti, specie utilizzando marcatori dotati di maggiore potere diagnostico e prognostico rispetto alla creatinina sierica, quali NGAL, Cistatina C e KIM-1.

Parole Chiave. CIN, AKI, Mezzo di contrasto iodato, GFR

\section{Indirizzo degli Autori:}

Prof. Michele Buemi

Cattedra di Nefrologia, Università di Messina

Dipartimento di Medicina Interna

UOC Terapia Intensiva e Tecniche Dialitiche

Via Consolare Valeria 1

Messina

Buemim@unime.it

\section{Bibliografia}

1. McCullough PA, Sandberg KR. Epidemiology of contrast-induced nephropathy. Rev Cardiovasc Med 2003; 4(Suppl 5): S3-S9.

2. Bolignano D, Donato V, Coppolino G, Campo S, Buemi A, Lacquaniti A, Buemi M. Neutrophil gelatinaseassociated lipocalin (NGAL) as a marker of kidney damage. Am J Kidney Dis 2008; 52: 595-605.

3. Weisbord SD, Palevsky PM. Prevention of contrastinduced nephropathy with volume expansion. Clin J Am Soc Nephrol 2008, 3(1): 273-80.

4. Hoffmann U, Fischereder M, Kruger B, Drobnik $\mathrm{W}$, Kramer BK. The value of $\mathrm{N}$-acetylcysteine in the prevention of radiocontrast agent-induced nephropathy seems questionable. J Am Soc Nephrol 2004; 15: 407-10. 